\title{
Ocean energy technology innovation development and application
}

\author{
demonstration
}

\author{
LI De- tang, Chen Shu-kun \\ (Ship and Marine ocean Engineering College of Zhejiang Ocean \\ University,Zhoushan316022,China) \\ (Shipping and Port Construction Engineering College of Zhejiang Ocean University, \\ Zhoushan316022, China)
}

Key Words: bad environment; wave energy; water; cage

Abstract: It is hard to imagine for people when they face the difficulty in Ocean energy development. how to access to abundant reserves of wave under harsh environmental conditions review of sea conditions, ancient China In flood control and management in the process of water, ancient China weren't fear of floods monstrous, a countless brilliant they created, is worth using for reference. This paper reviews the technical invention of the waterwheel, it combined with the author's research and innovation, the wave energy power research achievements will be applied in deep water net cage farming, industry development mode of integration, effectively promote the sustainable development of China's marine energy .

\section{Introduction}

\section{The urgency of the development of the ocean}

At present, China's energy production and consumption volume were has been ranked the forefront of the world, but in the supply and utilization of energy, the outstanding problems is the unreasonable structure of energy, renewable energy development and utilization ratio is low, the traditional extensive mode of economic development, is facing a shortage of resources exhaustion of bottlenecks and serious environmental pollution problems.

Therefore, in the development of the world's energy, people enter a new round of strategic adjustment period, it is very urgent and necessary for we actively to explore the ocean energy development and industrialization way.

\section{Key problems in the development of wave energy}

In Wave energy development, the key problem we faced is power generation equipment to withstand the storm impact test in the complex and harsh sea conditions (as shown in Figure 1); in particular, the wave energy is not continuous, it is very unstable, it requires the output power stable and no fluctuations to avoid the impact load bringing destruction to the user terminal; it needs test in withstand time for months, to get rid of the influence of seawater corrosion and creatures of the sea, power generation equipment is easy to maintain, safe and high reliable. 


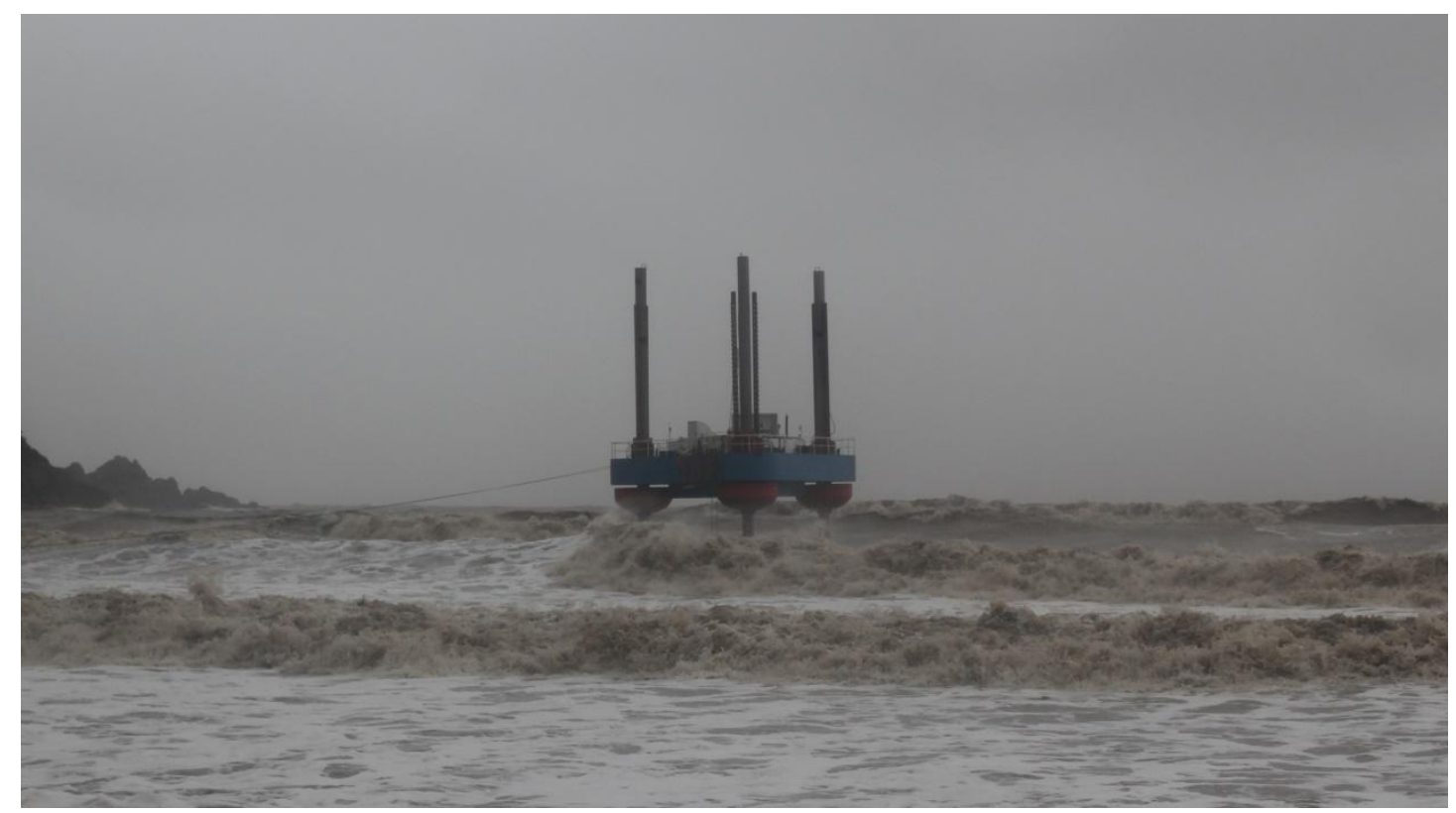

Fig.1 the self-protection status of 'Haiyuan1'in typhoon

In this paper, through the open today, combining the advantages of Zhou Shan sea farming, in the summary of the research and innovative results, the wave energy power generation and deep-water net cage integration of industry development mode, it can effectively promote the sustainable development of China's marine energy.

\section{Tankers Kam}

\section{Tankers of literary praise}

The eighty or ninety years since the last century, although the traditional waterwheel gradually disappeared from the fields, people forgot the beautiful scenery like a waterwheel, Tao's idyllic life paintings of landscapes (Figure 2). Water into from storage reservoirs, headrace power system. The sluice gate, hydraulic car, milling disc cleverly combined an original grain processing apparatus. Around these links, or built cobbled embankment, or planting was easy to grow willows, or put up a simple thatched pavilion, or built a brick house. The Xiao nan in" hometown the waterwheel to praise: I love cars > independent field is not afraid of defying heat cold exposed to wind and rain, appearance, I love cars can't stop moving, stop to the pursuit, I love cars good by the river, and good overall strength, good application to crops character [1-2]. 


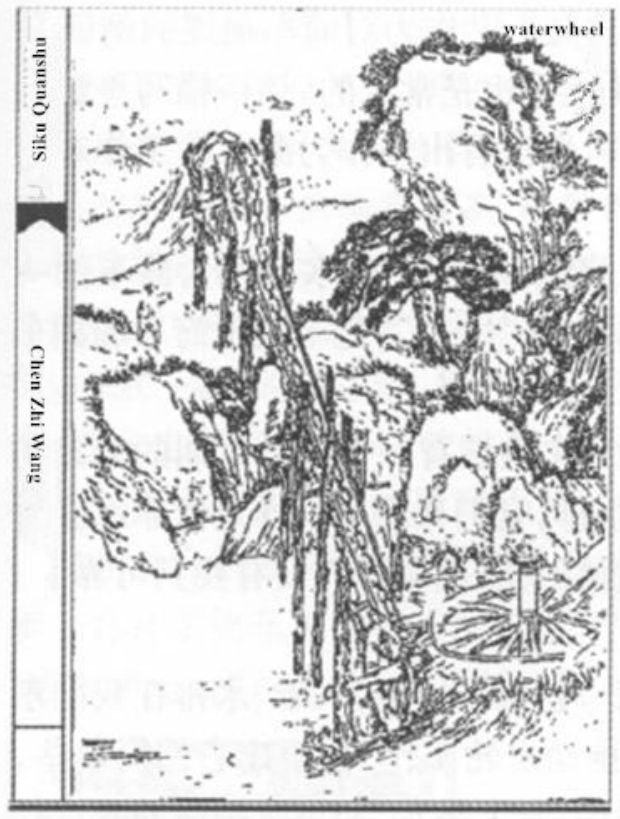

Fig. 2 ancient scroll wheel

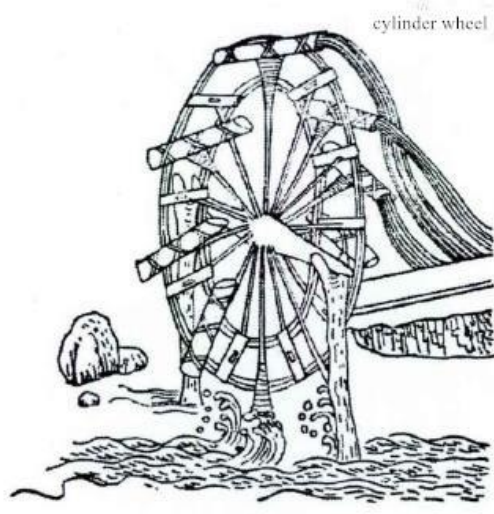

Fig. 3 cylinder cars

\section{Leading the world's ancient renewable energy revolution [2-3]}

The floods is the most brutal killer, but in ancient China people use the natural, they use the natural to transform process, from living by the water, afraid of the water, water to water, water worship, water pipe, created a brilliant. Among them, how to effectively extract water from low to high, waterwheel is one of the most successful inventions. It is the most typical and cars overturned, the energy conversion forms a linear motion, a circular movement.

(1) cylinder wheel

With hydraulic power impulse turbine automatic operation and carrying water, bamboo water poured into the sink in the automatic transmission, the hydraulic diameter is almost the same in high water (Figure 3).

(2) the water turns over

Hydro has vertical and horizontal .It is in the water gap, using hydraulic drive wood gear to rotate the rollover irrigation farming tools, applicable to low head hydraulic conditions (Figure 4)

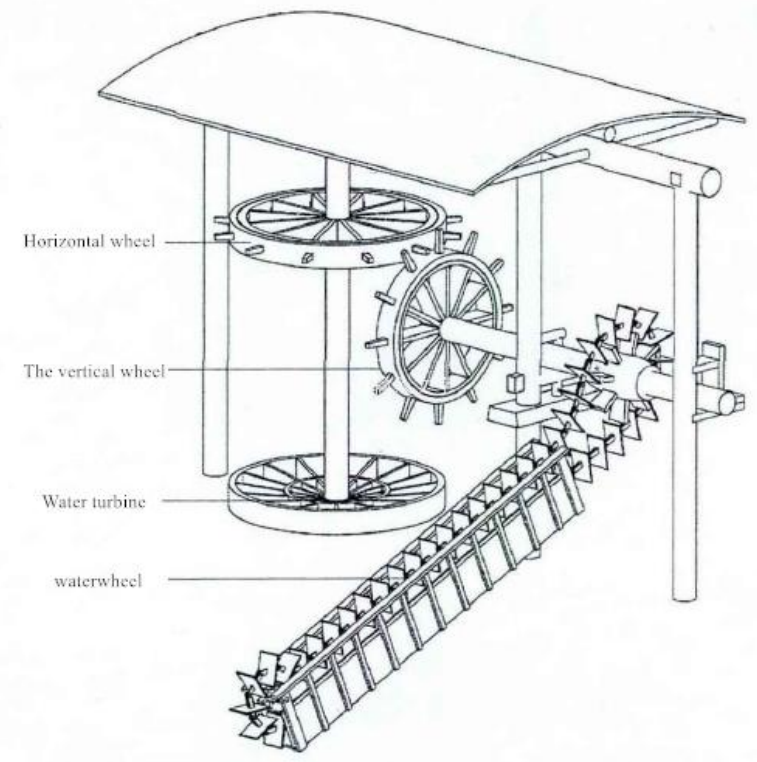

Fig.4 rollover 


\section{(3) The regional characteristics of water}

For a thousand years, the main reason of long lasting waterwheel is the distinctive regional style; it is suitable for complex environment in different conditions, to adapt to the regional differences of the natural conditions and resources. Especially in Lanzhou water tankers (Figure 5), for extraction of water from the Yellow River, only the wheel diameter tall to withstand the water of the Yellow River the strong impact force. In order to survive production, our fathers brought forth from the local natural conditions of, the invention of implement and utilization of the very full.

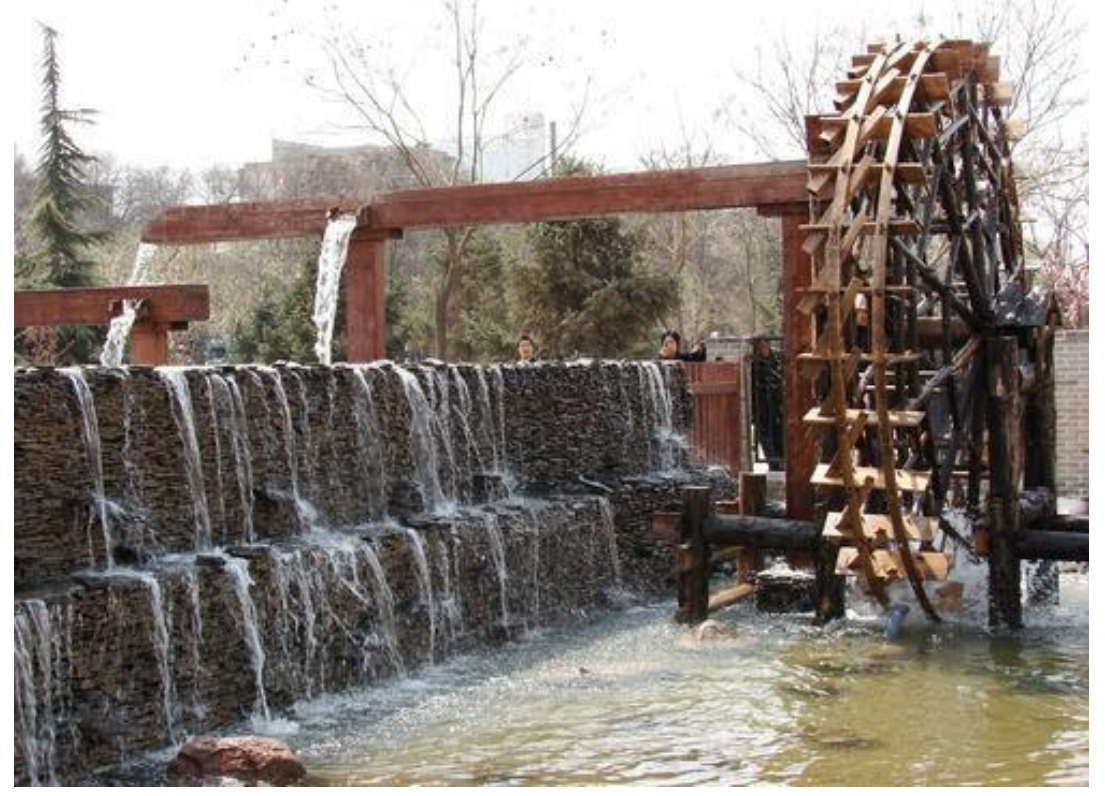

Fig.5 Lanzhou water tankers

\section{A contemporary enlightenment}

Waterwheel not only reflects the outstanding achievements of Chinese traditional farming tools, energy utilization in the traditional Chinese science and technology to the limit, but also the ancient Chinese science and technology .It is advanced and frontier, the coordination and unity of human and nature.

However, in this paper, the marine aquaculture compared with the ancients is still unsatisfactory. Mari culture in China are mainly concentrated in the $\mathrm{M} \sim 10 \mathrm{~m}$ shallow bays, beaches and coastal waters, utilization rate reached more than $90 \%$, and 10meters to 20meters isobaths increase breeding and utilization of less than $10 \%$, a serious imbalance in the distribution; although the harbor area accounted for only 30\% of the Mari culture area, production has accounted for aquaculture production more than $60 \%$. More due to the one-sided pursuit of economic benefits, fish and shrimp high density culture and layout, to the neglect of the long-term and environmental benefits, The development of local sea areas in our country is excessive, which is beyond the capacity of the capacity, the environment pollution is aggravated, the disease is rampant, and the germplasm degradation and so on, which has seriously restricted the sustainable development of the marine aquaculture industry.

In contrast, in the energy development, the ancients tried to use of hydropower development, to serve themselves. Placement of a hydraulic machinery often and do some work, such as the Barrell car shaft sometimes attached shuidui and Shuimo, multi-use, and as in the absence of natural rapids, dam water jet, forming rapids. The harmony with the natural ecological environment as a whole, innovation, in line with the contemporary national advocacy of sustainable development concept, to our oceans today can develop, rich profound scientific significance [3-4]. 


\section{Science and technology innovation of wave energy power generation at 'Haiyuan1'}

Aiming at the complex and harsh sea conditions and wave power existing technical problems, In the National Bureau of Oceanography, Ocean Energy Technology Center, Zhejiang Province and Zhou Shan city government leaders at all levels of care and support, Zhejiang Ocean University through technical research, developed a set of self-elevating wave power generation platform 'Haiyuan1' successfully, they broke through several key technical problems in the field of wave power generation.

\section{An adaptive random wave rack}

Real time tide changes caused the biggest problem, in order to ensure absorption type wave power generator normal operation, it need for frequent adjustment of buoy floating state and equilibrium position. The Adaptive Servo wave rack and a buoy, a hydraulic mechanism connected to the first wave of real-time collecting and transmitting and even tide changes up to 4 meters, it can also work normally. To effectively solve the problems caused by large variations in tidal power are discontinuous, to achieve all-weather continuous power.

\section{A two body double lift adjusting technique is invented.}

(1) The state of power, the main part of the platform to rise from the sea, only to float in the water fluctuation;

(2) The bad sea conditions, the float can also rise from the sea surface; resist the impact of the storm effectively;

(3) When the shift and fixation of the sea is carried out by ballast water injection, the insertion and pulling out piles are completed by itself.

Compared with similar technology of anchored, piling offshore operation: "a huge amount of construction, the cost of high status", the technical operation procedure is simple and reliable, and does not need the large-scale auxiliary operation equipment of the sea, and the production cost is reduced. At the same time, the core equipment of wave power generation is above sea level; it also eliminates the influence of seawater corrosion, increases the service life of equipment, and effectively reduces the cost of management and maintenance.

\section{A group of hydraulic cylinder energy technology is developed.}

By symmetrically arranged group of hydraulic cylinder and wave rack is meshed to overcome the float is only connected with a hydraulic cylinder to defects, which can fully absorb the wave energy, improve conversion efficiency, but also eliminate the wave disturbance on the impact. It realizes the mechanical energy to the hydraulic energy of the second level of smooth conversion, Figure 6 for the wave power generation hydraulic system schematic, a total of three sets of hydraulic ram system, and each group has eight hydraulic cylinders. 


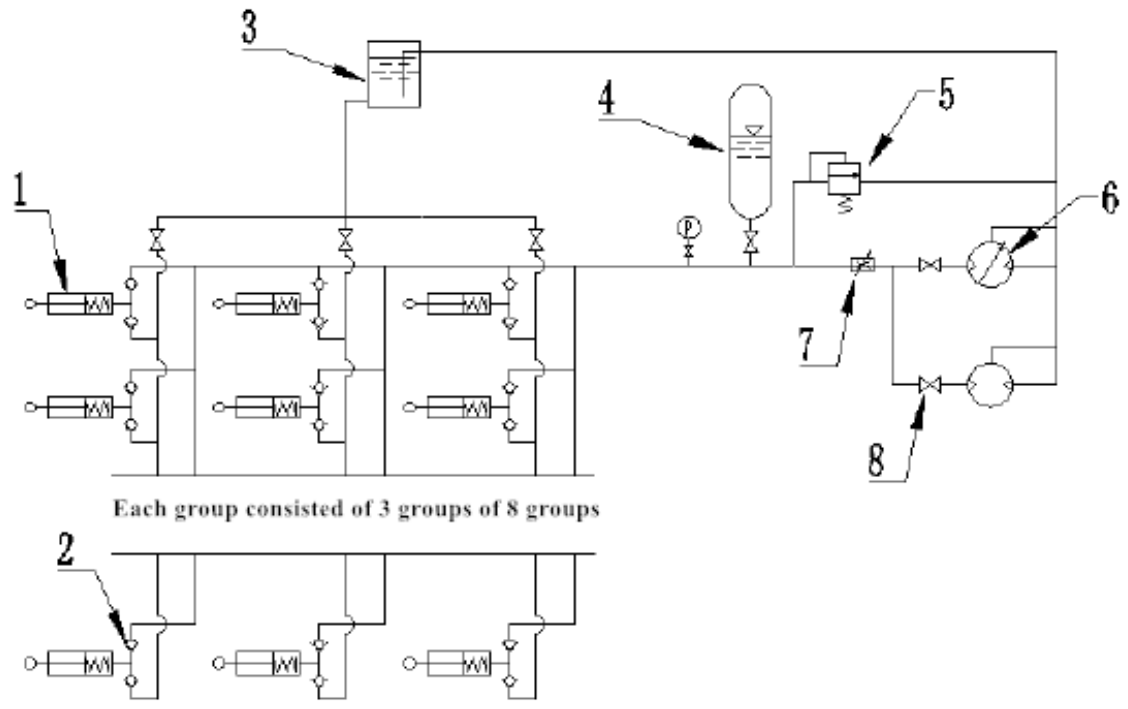

1 one-way hydraulic cylinder; 2 way valve; 3 hydraulic oil tank; 4 hydraulic -accumulator;

5 relief valve; 6 hydraulic motor; 7 speed control valve; 8 stop valve

Fig. 6 schematic diagram of the hydraulic system of wave power generation

A wave energy storage and automatic adjustment mechanism is invented.

On the basis of the innovative design of the system and the power system of hydraulic pumped storage, the different sizes of the wave energy to be stored and improves the energy conversion efficiency, and at the same time, it can automatically switch power output load, waves load output, Konami small load output, avoiding any loss caused by electric shocks, the third stage hydraulic energy to electrical energy is continuous and stable output of the transformation and Figure 7-9 test results for the system power [5].

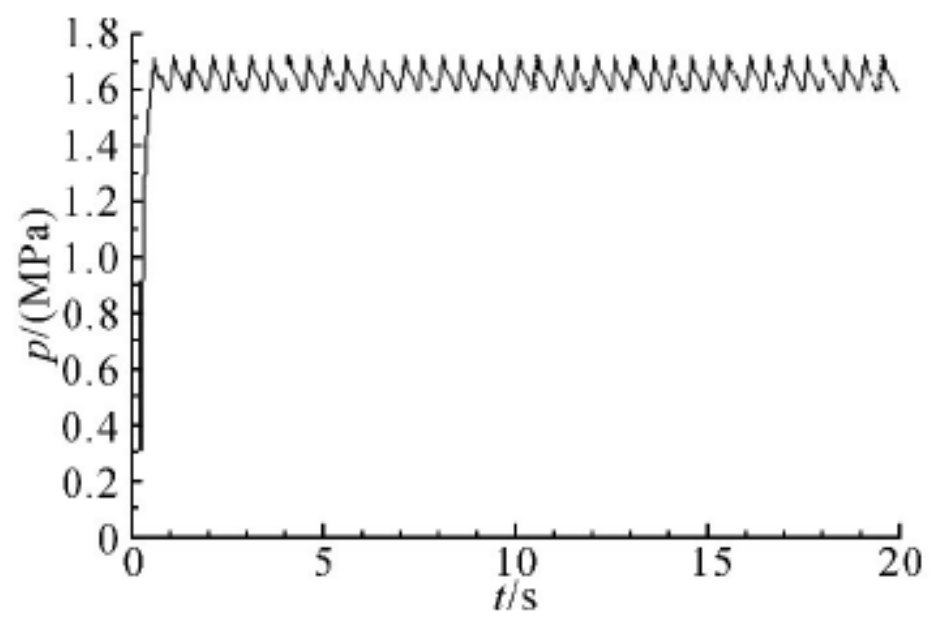

Fig.7 output pressure of hydraulic system 


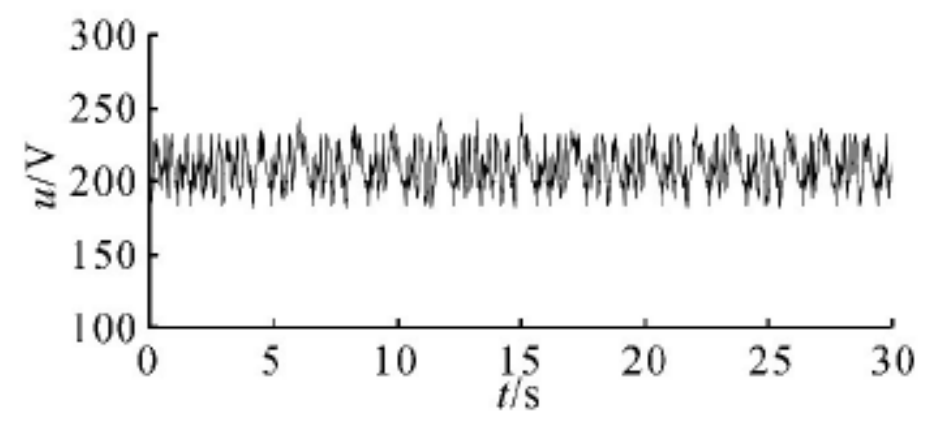

Fig.8 output voltage measurement curve of power generation system

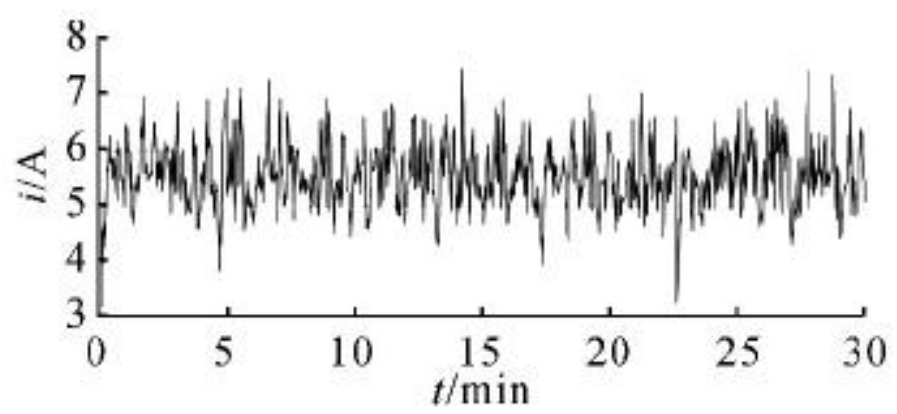

Fig.9 output current measurement curve of power generation system

\section{Sea trials and the results of the transformation direction}

\section{Sea trials}

In April 6,2013 "Haiyuan1" wave power generation platform by the shipyard drag airlines to Zhou Shan ZhuJiajian DongSha sea area in place of power, the application of the real sea proved that wave power device technology is mature, reliable operation, not only to adapt to the complex marine environment, adverse conditions can achieve self-protection, and all of the core equipment without affecting seawater corrosion, has withstood the ultra-strong typhoon "Su", strong tropical storm, "Mei Tan", "Buffett attack tests.

\section{Achievements in the direction of transformation}

"Haiyuan1" wave power generation platform, that it has very good prospects, combined with cage technology, its anti-storm ability, can effectively solve the offshore aquaculture pollution and deep water aquaculture anti wave key technical problems, not only to provide clean energy for farms, meet the net change cleaning, automatic feeding, breeding monitoring power demand, and break the cage wave of harassment, can form a set of advanced offshore power station, set power generation, production and life, and feed on the net for rest in one, has good technical and economic value and market prospects for the healthy and rapid development of cage culture and scientific management.

\section{Summary}

Under the background of the global energy system transformation, accelerating the deterioration of the ecological environment and the increasing pressure on the response to climate change, general secretary Xi Jinping particular emphasis on energy security is a national economic and social development of the overall situation, strategic issues, it is vital to the prosperity and development of 
the country, the improvement of the people's life and the social security of the society. Without innovation, scientific research and technological development will always follow and imitate heteronomy. Therefore, through the ocean energy technology innovation and demonstration application, "Haiyuan1"wavepower generation platform transformation in deep water net cage farming, based on the national conditions of our country, will accelerate to promote China's energy production and utilization of change.

\section{Reference}

[1] Xiao Nan. Home Waterwheel [J] Hunan literature home2016, 03:145-147.

[2] Li, Genpan. The origin and development of waterwheel (Volume I) [J].Agricultural history of China.2011, 02:3-18.

[3] Fang, Lisong. Study on the traditional Chinese waterwheel [D].Nanjing: Nanjing Agricultural University, 2010

[4] Wang, Dongshi, Gao, Jinyu. Development and present situation of seawater aquaculture in China [J].Chinese aquatic.2015, 04:39-42.

[5] Lu Qin, Li De-tang, etc. Design of oscillating buoy wave power generation system based on hydraulic transmission [J].Journal of Zhejiang University ((Engineering Science Edition).2016, 02:234-240. 